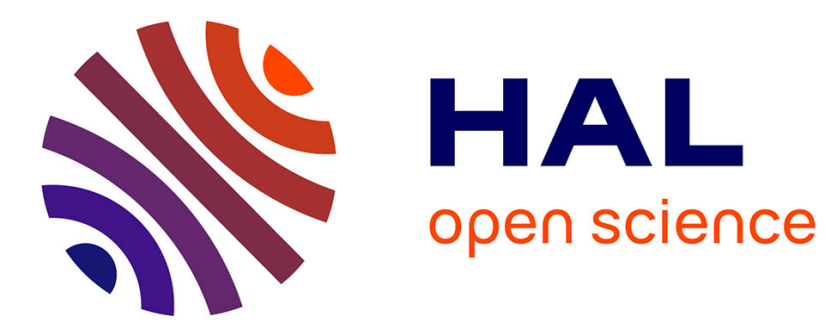

\title{
Strong solutions for a 1D viscous bilayer Shallow Water model
}

\author{
Jean de Dieu Zabsonré, Carine Lucas, Adama Ouedraogo
}

\section{To cite this version:}

Jean de Dieu Zabsonré, Carine Lucas, Adama Ouedraogo. Strong solutions for a 1D viscous bilayer Shallow Water model. Nonlinear Analysis: Real World Applications, 2013, 14 (2), pp.1216 - 1224. 10.1016/j.nonrwa.2012.09.012 . hal-00664215v2

\section{HAL Id: hal-00664215 \\ https://hal.science/hal-00664215v2}

Submitted on 7 Feb 2013

HAL is a multi-disciplinary open access archive for the deposit and dissemination of scientific research documents, whether they are published or not. The documents may come from teaching and research institutions in France or abroad, or from public or private research centers.
L'archive ouverte pluridisciplinaire HAL, est destinée au dépôt et à la diffusion de documents scientifiques de niveau recherche, publiés ou non, émanant des établissements d'enseignement et de recherche français ou étrangers, des laboratoires publics ou privés. 


\title{
Strong solutions for a 1D viscous bilayer Shallow Water model
}

\author{
Jean De Dieu ZaBSONRÉ* \\ ISEA, Université Polytechnique de Bobo-Dioulasso, 01 BP 1091 Bobo-Dioulasso 01, \\ Burkina Faso \\ Carine LUCAS \\ MAPMO UMR CNRS 7349, Université d'Orléans, Bâtiment de mathématiques, B.P. \\ 6759 - 45067 Orléans cedex 2, France \\ Adama Ouedraogo \\ ISEA, Université Polytechnique de Bobo-Dioulasso, 01 BP 1091 Bobo-Dioulasso 01, \\ Burkina Faso
}

\begin{abstract}
In this paper, we consider a viscous bilayer shallow water model in one space dimension that represents two superposed immiscible fluids. For this model, we prove the existence of strong solutions in a periodic domain. The initial heights are required to be bounded above and below away from zero and we get the same bounds for every time. Our analysis is based on the construction of approximate systems which satisfy the BD entropy and on the method developed by A. Mellet and A. VAsseur to obtain the existence of global strong solutions for the one dimensional Navier-Stokes equations.

Keywords: Strong solutions, shallow water, viscous models, bilayer, stability.

2000 MSC: 35Q35

*Corresponding author

Email addresses: jzabsonre@gmail.com (Jean De Dieu ZABSONRÉ), carine.lucas@univ-orleans.fr (Carine LUCAS), adam_ouedraogo3@yahoo.fr (Adama OUEDRAOGO)
\end{abstract}




\section{Introduction.}

In this paper, we study the global existence in time of strong solutions to the following viscous bilayer shallow water model:

$$
\begin{aligned}
& \partial_{t} h_{1}+\partial_{x}\left(h_{1} v_{1}\right)=0, \\
& \partial_{t}\left(h_{1} v_{1}\right)+\partial_{x}\left(h_{1} v_{1}^{2}\right)+g h_{1} \partial_{x} h_{1}+r g h_{1} \partial_{x} h_{2}-\nu_{1} \partial_{x}\left(h_{1} \partial_{x} v_{1}\right)=0, \\
& \partial_{t} h_{2}+\partial_{x}\left(h_{2} v_{2}\right)=0, \\
& \partial_{t}\left(h_{2} v_{2}\right)+\partial_{x}\left(h_{2} v_{2}^{2}\right)+g h_{2} \partial_{x} h_{2}+g h_{2} \partial_{x} h_{1}-\nu_{2} \partial_{x}\left(h_{2} \partial_{x} v_{2}\right)=0 .
\end{aligned}
$$

where $(t, x) \in(0, T) \times \Omega$, and $\Omega$ is a periodic domain in one dimension. These equations represent a system composed of two layers of immiscible fluids. Index 1 refers to the deeper layer and index 2 to the upper layer, see Figure 1.

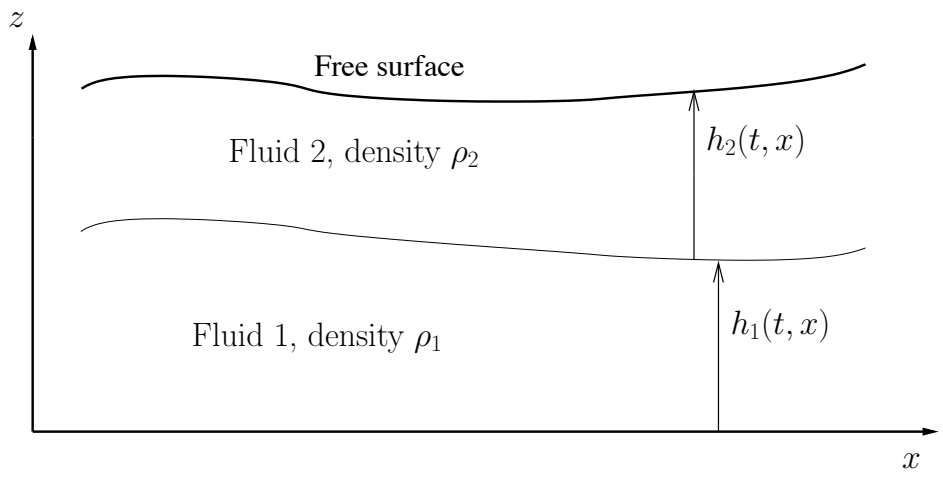

Figure 1: Notations for the bilayer model

We denote by $\rho_{1}$ and $\rho_{2}$ the densities of each layer of fluid, and $r$ is their ratio $r=\rho_{2} / \rho_{1}<1$. The quantities $\nu_{1}$ and $\nu_{2}$ are the respective kinematic viscosity, that is $\nu_{i}=\mu_{i} / \rho_{i}$ where $\mu_{i}$ is the dynamic viscosity. This model is formally derived in [1] in the two dimensional case. Such a model appears naturally in geophysical flows, see for example $[2,3]$.

Let us give a non-exhaustive list of the results on the existence of solutions to equations describing the motion of fluid substances. The existence of solutions to the one dimensional Navier-Stokes equations (which includes the shallow water equations) has been studied by many authors. In the case of a constant viscosity, the existence of weak solutions was first studied in [4] 
with smooth initial data close to equilibrium. The case of discontinuous data was solved in $[5,6,7,8]$. In particular, in [7], D. HofF proved the existence of weak solutions with large discontinuous initial data that can have different limits at $x= \pm \infty$. This theorem requires the initial data to be bounded away from zero. The first global existence result for initial density which can vanish was shown in [9]. This result was extended later in [10] to the full compressible Navier-Stokes equations.

More recently, in $[11,12,13]$, D. Bresch and B. Desjardins obtained the existence of a global weak solution for a 2D shallow water and a Korteweg systems with a diffusion term of the form $\operatorname{div}(h D(u))$. The key point that gave them this result is an entropy inequality namely BD entropy. This BD entropy has been extended in [14] to more general Navier-Stokes equations with an algebraic relation between the shear and the bulk viscosity coefficients. The authors used quadratic friction terms and capillary effects to get the stability of the system. In [15], using this inequality again, A. MELLET and A. VASSEUR obtained the stability of global weak solutions for the compressible isentropic Navier-Stokes equations without any regularizing term. Another consequence of this BD entropy is that it gives control on some negative power of the density in one dimension. This nice control was shown in [16] and led the authors to prove that vacuum cannot arise if there is no vacuum at the initial time. With this result, they obtained the existence of strong solutions for the one dimensional viscous compressible Navier-Stokes equations. However, the admissible viscosity must be some power of the density, which does not take the shallow water system into account.

Let us go back to the shallow water equations, and more precisely to bilayer systems. An existence result on a one dimensional bilayer shallow water model was studied in [17]. The authors obtained the existence, uniqueness and some smoothness of weak solutions under the assumption that the data are sufficiently small. However the bilayer shallow water they considered is not exactly the one we study here. In this paper, we are interested in another system of bilayer immiscible fluid obtained by derivation in [1]. These equations have been studied in $[18,19]$; the authors proved the existence of a global weak solution for viscous bilayer Shallow Water models with the BD inequality (but in two dimensions).

This paper deals with strong solutions, in one dimension. The main existence result is stated in Section 2. To prove this statement, we construct approximate solutions in Section 3 following the work performed in [20, 9], based on [21]. Indeed in [20], the authors obtained the existence of strong so- 
lutions for the one dimensional compressible Navier-Stokes equations, which include the case of shallow water. They proved that there exists some time after which the density is bounded from below by a positive constant and the weak solution becomes a strong one. Here, we prove that our approximate system verifies the BD entropy, which gives the lower bound for the water heights. Next, in Section 4, we obtain the existence of strong solutions for the approximate system by using the regularity theorem for smooth data given in [22], and we pass to the limit. The last part (Appendix A) is dedicated to the proofs of the classical energy and $\mathrm{BD}$ entropy inequalities.

\section{Main result}

Theorem 2.1. Let the initial data $h_{i_{0}}=h_{i \mid t=0}$ and $v_{i_{0}}=v_{i \mid t=0}$ satisfy the conditions

$$
\begin{gathered}
0<\underline{c}_{0} \leq h_{i_{0}} \leq \bar{c}_{0}, \\
h_{i_{0}} \in H^{1}(\Omega), \quad v_{i_{0}} \in H^{1}(\Omega),
\end{gathered}
$$

where $\underline{c}_{0}$ and $\bar{c}_{0}$ are some positive constants. Assume that the viscosities $\nu_{1}$ and $\nu_{2}$ verify the relation

$$
\nu_{1}>\frac{r\left(\nu_{1}+\nu_{2}\right)}{2}(2-\alpha), \quad \nu_{2}>\frac{\nu_{1}+\nu_{2}}{2}(2-\alpha)
$$

with $1>\alpha>2 r /(1+r)$. Then the problem (1)-(4) admits a strong solution $\left(h_{1}, h_{2}, v_{1}, v_{2}\right)$ such that $h_{i} \in L^{\infty}\left(0, T ; H^{1}(\Omega)\right), v_{i} \in L^{\infty}\left(0, T ; H^{1}(\Omega)\right) \cap$ $L^{2}\left(0, T ; H^{2}(\Omega)\right)$ and $\partial_{t} v_{i} \in L^{2}\left(0, T ; L^{2}(\Omega)\right)$.

To prove this theorem, we construct approximate smooth solutions in the next section, following the work performed in [20].

\section{Approximate smooth solutions}

In this section, we consider the approximate system defined as follows:

$$
\begin{aligned}
& \partial_{t} h_{1, \varepsilon}+\partial_{x}\left(h_{1, \varepsilon} v_{1, \varepsilon}\right)=0, \\
& \partial_{t}\left(h_{1, \varepsilon} v_{1, \varepsilon}\right)+\partial_{x}\left(h_{1, \varepsilon} v_{1, \varepsilon}^{2}\right)+g h_{1, \varepsilon} \partial_{x} h_{1, \varepsilon} \\
& \quad+r g h_{1, \varepsilon} \partial_{x} h_{2, \varepsilon}-\nu_{1} \partial_{x}\left(\psi_{\varepsilon}\left(h_{1, \varepsilon}\right) \partial_{x} v_{1, \varepsilon}\right)=0, \\
& \partial_{t} h_{2, \varepsilon}+\partial_{x}\left(h_{2, \varepsilon} v_{2, \varepsilon}\right)=0 \\
& \partial_{t}\left(h_{2, \varepsilon} v_{2, \varepsilon}\right)+\partial_{x}\left(h_{2, \varepsilon} v_{2, \varepsilon}^{2}\right)+g h_{2, \varepsilon} \partial_{x} h_{2, \varepsilon} \\
& \quad+g h_{2, \varepsilon} \partial_{x} h_{1, \varepsilon}-\nu_{2} \partial_{x}\left(\psi_{\varepsilon}\left(h_{2, \varepsilon}\right) \partial_{x} v_{2, \varepsilon}\right)=0 .
\end{aligned}
$$


where $\psi_{\varepsilon}\left(h_{i, \varepsilon}\right)=h_{i, \varepsilon}+\varepsilon h_{i, \varepsilon}^{\beta}($ for $i=1,2), \varepsilon$ is a small parameter satisfying

$$
\varepsilon<\frac{1-r}{1+r}
$$

$\beta$ belongs to $(0,1 / 2)$. We also assume the following relation on the viscosities:

$$
\nu_{1}>\frac{r\left(\nu_{1}+\nu_{2}\right)}{2}(1+\varepsilon), \quad \nu_{2}>\frac{\nu_{1}+\nu_{2}}{2}(1+\varepsilon) .
$$

This type of approximate systems can be constructed by a mollifying process as in $[23,7]$. We suppose that the initial data $h_{i, \varepsilon \mid t=0}=h_{i_{0}, \varepsilon}, \quad h_{i, \varepsilon} v_{i, \varepsilon \mid t=0}=$ $h_{i_{0}, \varepsilon} v_{i_{0}, \varepsilon} \in \mathcal{C}(\bar{\Omega})$ satisfy $(5)$ and that:

$$
h_{i_{0}, \varepsilon} \rightarrow h_{i_{0}} \text { in } L^{1}(\Omega), \quad h_{i_{0}, \varepsilon}^{1 / 2} \rightarrow h_{i_{0}}^{1 / 2} \text { in } H^{1}(\Omega), \quad h_{i_{0}, \varepsilon} v_{i_{0}, \varepsilon}^{2} \rightarrow h_{i_{0}} v_{i_{0}}^{2} \text { in } L^{1}(\Omega) .
$$

Remark 3.1. Let us define $\varphi_{\varepsilon}\left(h_{i, \varepsilon}\right)=h_{i, \varepsilon}+\frac{\varepsilon}{\beta} h_{i, \varepsilon}^{\beta}$. Then we have

$$
h_{i, \varepsilon} \varphi_{\varepsilon}^{\prime}\left(h_{i, \varepsilon}\right)=\psi_{\varepsilon}\left(h_{i, \varepsilon}\right)
$$

This relation will be used to get the BD entropy, see Appendix $A$.

We assume that the initial energy associated to the approximate system (6)-(9) is taken such that

$$
\begin{aligned}
\mathcal{E}_{0}=\frac{1}{2} \int_{\Omega} h_{1_{0}, \varepsilon}\left|v_{1_{0}, \varepsilon}\right|^{2}+\frac{r}{2} \int_{\Omega} h_{2_{0}, \varepsilon}\left|v_{2_{0}, \varepsilon}\right|^{2} & +\frac{g(1-r)}{2} \int_{\Omega}\left|h_{1_{0}, \varepsilon}\right|^{2} \\
& +\frac{r g}{2} \int_{\Omega}\left|h_{1_{0}, \varepsilon}+h_{2_{0}, \varepsilon}\right|^{2} \leq C \varepsilon^{2} \leq C
\end{aligned}
$$

and

$$
\mathcal{F}_{0}=\frac{1}{2} \int_{\Omega}\left|\nu_{1} \frac{\partial_{x} \varphi_{\varepsilon}\left(h_{1_{0}, \varepsilon}\right)}{\sqrt{h_{1_{0}, \varepsilon}}}\right|^{2}+\frac{1}{2} \int_{\Omega}\left|\nu_{2} \frac{\partial_{x} \varphi_{\varepsilon}\left(h_{2_{0}, \varepsilon}\right)}{\sqrt{h_{2_{0}, \varepsilon}}}\right|^{2} \leq C \varepsilon^{2} \leq C
$$

for $\varepsilon>0$ small enough. 


\subsection{Energy inequalities}

In this section, we give the classical energy inequality and the BD entropy for the solution of System (6)-(9).

Proposition 3.2. If $\left(h_{1, \varepsilon}, h_{2, \varepsilon}, v_{1, \varepsilon}, v_{2, \varepsilon}\right)$ is a smooth solution of System (6)(9), then the following classical inequality holds:

$$
\begin{gathered}
\frac{1}{2} \frac{d}{d t} \int_{\Omega} h_{1, \varepsilon}\left|v_{1, \varepsilon}\right|^{2}+\frac{r}{2} \frac{d}{d t} \int_{\Omega} h_{2, \varepsilon}\left|v_{2, \varepsilon}\right|^{2} \\
\quad+\nu_{1} \int_{\Omega} \psi_{\varepsilon}\left(h_{1, \varepsilon}\right)\left(\partial_{x} v_{1, \varepsilon}\right)^{2} d x+r \nu_{2} \int_{\Omega} \psi_{\varepsilon}\left(h_{2, \varepsilon}\right)\left(\partial_{x} v_{2, \varepsilon}\right)^{2} d x \\
\quad+\frac{g(1-r)}{2} \frac{d}{d t} \int_{\Omega}\left|h_{1, \varepsilon}\right|^{2}+\frac{r g}{2} \frac{d}{d t} \int_{\Omega}\left|h_{1, \varepsilon}+h_{2, \varepsilon}\right|^{2} \leq 0 .
\end{gathered}
$$

Corollary 3.3. The classical energy estimate gives the following uniform bounds:

$$
\begin{aligned}
& \left\|\sqrt{h_{i, \varepsilon}} v_{i, \varepsilon}\right\|_{L^{\infty}\left(0, T ; L^{2}(\Omega)\right)} \leq C(T) ; \quad \\
& \left\|\sqrt{\psi_{\varepsilon}\left(h_{i, \varepsilon}\right)} \partial_{x} v_{i, \varepsilon}\right\|_{L^{2}\left(0, T ; L^{2}(\Omega)\right)} \leq C(T) .
\end{aligned}
$$

As these bounds are not informative enough to get the existence of solutions, we write the BD entropy to have more information on the solution.

Proposition 3.4. Let $\left(h_{1, \varepsilon}, h_{2, \varepsilon}, v_{1, \varepsilon}, v_{2, \varepsilon}\right)$ be a smooth solution of $(6)-(9)$, then the following mathematical BD entropy inequality holds:

$$
\begin{aligned}
\frac{1}{2} \frac{d}{d t} \int_{\Omega} h_{1, \varepsilon} \mid v_{1, \varepsilon} & +\left.\nu_{1} \frac{\partial_{x} \varphi_{\varepsilon}\left(h_{1, \varepsilon}\right)}{h_{1, \varepsilon}}\right|^{2}+\frac{r}{2} \frac{d}{d t} \int_{\Omega} h_{2, \varepsilon}\left|v_{2, \varepsilon}+\nu_{2} \frac{\partial_{x} \varphi_{\varepsilon}\left(h_{2, \varepsilon}\right)}{h_{2, \varepsilon}}\right|^{2} \\
& +\frac{g(1-r)}{2} \frac{d}{d t} \int_{\Omega}\left|h_{1, \varepsilon}\right|^{2}+\frac{r g}{2} \frac{d}{d t} \int_{\Omega}\left|h_{1, \varepsilon}+h_{2, \varepsilon}\right|^{2} \\
& +\nu_{1} g \int_{\Omega} \varphi_{\varepsilon}^{\prime}\left(h_{1, \varepsilon}\right)\left(\partial_{x} h_{1, \varepsilon}\right)^{2}+\nu_{2} r g \int_{\Omega} \varphi_{\varepsilon}^{\prime}\left(h_{2, \varepsilon}\right)\left(\partial_{x} h_{2, \varepsilon}\right)^{2} \\
& +\nu_{1} r g \int_{\Omega} \partial_{x} h_{2, \varepsilon} \partial_{x} \varphi_{\varepsilon}\left(h_{1, \varepsilon}\right)+\nu_{2} r g \int_{\Omega} \partial_{x} h_{1, \varepsilon} \partial_{x} \varphi_{\varepsilon}\left(h_{2, \varepsilon}\right) \leq 0 .
\end{aligned}
$$


Corollary 3.5. Equation (18) can be integrated over $[0, t]$, for $t \in[0, T]$. Then we get:

$$
\begin{aligned}
& \frac{1}{2} \int_{\Omega} h_{1, \varepsilon}\left|v_{1, \varepsilon}+\nu_{1} \frac{\partial_{x} \varphi_{\varepsilon}\left(h_{1, \varepsilon}\right)}{h_{1, \varepsilon}}\right|^{2}+\frac{r}{2} \int_{\Omega} h_{2, \varepsilon}\left|v_{2, \varepsilon}+\nu_{2} \frac{\partial_{x} \varphi_{\varepsilon}\left(h_{2, \varepsilon}\right)}{h_{2, \varepsilon}}\right|^{2} \\
& \quad+\frac{g(1-r)}{2} \int_{\Omega}\left|h_{1, \varepsilon}\right|^{2}+\frac{r g}{2} \int_{\Omega}\left|h_{1, \varepsilon}+h_{2, \varepsilon}\right|^{2} \\
& \quad+\nu_{1} g \int_{0}^{t} \int_{\Omega} \varphi_{\varepsilon}^{\prime}\left(h_{1, \varepsilon}\right)\left(\partial_{x} h_{1, \varepsilon}\right)^{2}+\nu_{2} r g \int_{0}^{t} \int_{\Omega} \varphi_{\varepsilon}^{\prime}\left(h_{2, \varepsilon}\right)\left(\partial_{x} h_{2, \varepsilon}\right)^{2} \\
& \quad+\nu_{1} r g \int_{0}^{t} \int_{\Omega} \partial_{x} h_{2, \varepsilon} \partial_{x} \varphi_{\varepsilon}\left(h_{1, \varepsilon}\right)+\nu_{2} r g \int_{0}^{t} \int_{\Omega} \partial_{x} h_{1, \varepsilon} \partial_{x} \varphi_{\varepsilon}\left(h_{2, \varepsilon}\right) \\
& \leq \frac{1}{2} \int_{\Omega} h_{1_{0}, \varepsilon}\left|v_{1_{0}, \varepsilon}+\nu_{1} \frac{\partial_{x} \varphi_{\varepsilon}\left(h_{1_{0}, \varepsilon}\right)}{h_{1_{0}, \varepsilon}}\right|^{2}+\frac{r}{2} \int_{\Omega} h_{2_{0}, \varepsilon}\left|v_{2_{0}, \varepsilon}+\nu_{2} \frac{\partial_{x} \varphi_{\varepsilon}\left(h_{2_{0}, \varepsilon}\right)}{h_{2_{0}, \varepsilon}}\right|^{2} \\
& +\frac{g(1-r)}{2} \int_{\Omega}\left|h_{1_{0}, \varepsilon}\right|^{2}+\frac{r g}{2} \int_{\Omega}^{\left|h_{1_{0}, \varepsilon}+h_{2_{0}, \varepsilon}\right|^{2}}
\end{aligned}
$$

With the two relations (14) and (15), we can write:

$$
\begin{aligned}
& \frac{1}{2} \int_{\Omega} h_{1, \varepsilon}\left|v_{1, \varepsilon}+\nu_{1} \frac{\partial_{x} \varphi_{\varepsilon}\left(h_{1, \varepsilon}\right)}{h_{1, \varepsilon}}\right|^{2}+\frac{r}{2} \int_{\Omega} h_{2, \varepsilon}\left|v_{2, \varepsilon}+\nu_{2} \frac{\partial_{x} \varphi_{\varepsilon}\left(h_{2, \varepsilon}\right)}{h_{2, \varepsilon}}\right|^{2} \\
& \quad+\frac{g(1-r)}{2} \int_{\Omega}\left|h_{1, \varepsilon}\right|^{2}+\frac{r g}{2} \int_{\Omega}\left|h_{1, \varepsilon}+h_{2, \varepsilon}\right|^{2} \\
& \quad+\nu_{1} g \int_{0}^{t} \int_{\Omega} \varphi_{\varepsilon}^{\prime}\left(h_{1, \varepsilon}\right)\left(\partial_{x} h_{1, \varepsilon}\right)^{2}+\nu_{2} r g \int_{0}^{t} \int_{\Omega} \varphi_{\varepsilon}^{\prime}\left(h_{2, \varepsilon}\right)\left(\partial_{x} h_{2, \varepsilon}\right)^{2} \\
& \quad+\nu_{1} r g \int_{0}^{t} \int_{\Omega} \partial_{x} h_{2, \varepsilon} \partial_{x} \varphi_{\varepsilon}\left(h_{1, \varepsilon}\right)+\nu_{2} r g \int_{0}^{t} \int_{\Omega} \partial_{x} h_{1, \varepsilon} \partial_{x} \varphi_{\varepsilon}\left(h_{2, \varepsilon}\right) \\
& \leq c\left(\mathcal{E}_{0}+\mathcal{F}_{0}\right) \leq C \varepsilon^{2} .
\end{aligned}
$$

Propositions 3.2 and 3.4 are proved in Appendix A.

To obtain new bounds thanks to the BD entropy (in order to complete Corollary 3.3), it remains to control the last two terms of the left hand side of Equation (18). Since $\varphi_{\varepsilon}^{\prime}\left(h_{i, \varepsilon}\right)>1$ (for $i=1,2$ ), one can write:

$$
\begin{aligned}
Q & =\nu_{1} r g \int_{\Omega}\left|\partial_{x} h_{2, \varepsilon} \partial_{x} \varphi_{\varepsilon}\left(h_{1, \varepsilon}\right)\right|+\nu_{2} r g \int_{\Omega}\left|\partial_{x} h_{1, \varepsilon} \partial_{x} \varphi_{\varepsilon}\left(h_{2, \varepsilon}\right)\right| \\
& \leq \nu_{1} r g \int_{\Omega}\left|\partial_{x} \varphi_{\varepsilon}\left(h_{2, \varepsilon}\right) \partial_{x} \varphi_{\varepsilon}\left(h_{1, \varepsilon}\right)\right|+\nu_{2} r g \int_{\Omega}\left|\partial_{x} \varphi_{\varepsilon}\left(h_{1, \varepsilon}\right) \partial_{x} \varphi_{\varepsilon}\left(h_{2, \varepsilon}\right)\right|,
\end{aligned}
$$


and consequently

$$
Q \leq \frac{\nu_{1} r g+\nu_{2} r g}{2} \int_{\Omega}\left|\partial_{x} \varphi_{\varepsilon}\left(h_{1, \varepsilon}\right)\right|^{2}+\frac{\nu_{1} r g+\nu_{2} r g}{2} \int_{\Omega}\left|\partial_{x} \varphi_{\varepsilon}\left(h_{2, \varepsilon}\right)\right|^{2} .
$$

Next, we split the two integrals by using the sets $\left\{h_{i, \varepsilon} \leq 1\right\}$ and $\left\{h_{i, \varepsilon}>1\right\}$, with $i=1$ for the first integral and $i=2$ for the second one. Since $\beta<\frac{1}{2}$, $\varphi_{\varepsilon}^{\prime}\left(h_{i, \varepsilon}\right) \leq 1+\varepsilon$ in the set $\left\{h_{i, \varepsilon}>1\right\}$. Then, we have

$$
\begin{aligned}
Q \leq & \frac{\nu_{1} r g+\nu_{2} r g}{2} \int_{\left\{h_{1, \varepsilon} \leq 1\right\}}\left|\frac{\partial_{x} \varphi_{\varepsilon}\left(h_{1, \varepsilon}\right)}{\sqrt{h_{1, \varepsilon}}}\right|^{2} \\
& +\frac{\nu_{1} r g+\nu_{2} r g}{2} \int_{\left\{h_{1, \varepsilon}>1\right\}}(1+\varepsilon) \varphi_{\varepsilon}^{\prime}\left(h_{1, \varepsilon}\right)\left(\partial_{x}\left(h_{1, \varepsilon}\right)\right)^{2} \\
& +\frac{\nu_{1} r g+\nu_{2} r g}{2} \int_{\left\{h_{2, \varepsilon} \leq 1\right\}}\left|\frac{\partial_{x} \varphi_{\varepsilon}\left(h_{2, \varepsilon}\right)}{\sqrt{h_{2, \varepsilon}}}\right|^{2} \\
& +\frac{\nu_{1} r g+\nu_{2} r g}{2} \int_{\left\{h_{2, \varepsilon}>1\right\}}(1+\varepsilon) \varphi_{\varepsilon}^{\prime}\left(h_{2, \varepsilon}\right)\left(\partial_{x}\left(h_{2, \varepsilon}\right)\right)^{2} .
\end{aligned}
$$

The two quantities $\frac{\nu_{1} r g+\nu_{2} r g}{2} \int_{\left\{h_{1, \varepsilon}>1\right\}}(1+\varepsilon) \varphi_{\varepsilon}^{\prime}\left(h_{1, \varepsilon}\right)\left(\partial_{x}\left(h_{1, \varepsilon}\right)\right)^{2}$ and $\frac{\nu_{1} r g+\nu_{2} r g}{2} \int_{\left\{h_{2, \varepsilon}>1\right\}}(1+\varepsilon) \varphi_{\varepsilon}^{\prime}\left(h_{2, \varepsilon}\right)\left(\partial_{x}\left(h_{2, \varepsilon}\right)\right)^{2}$ can be merged with the left hand side of Equation (18). Due to conditions (11) on the viscosities and (10) on $\varepsilon$, the quantities $\left(\nu_{1}-\frac{r \nu_{1}+r \nu_{2}}{2}\right) g \int_{\Omega}(1+\varepsilon) \varphi_{\varepsilon}^{\prime}\left(h_{1, \varepsilon}\right)\left(\partial_{x}\left(h_{1, \varepsilon}\right)\right)^{2}$ and $\left(r \nu_{2}-\frac{r \nu_{1}+r \nu_{2}}{2}\right) g \int_{\Omega}(1+\varepsilon) \varphi_{\varepsilon}^{\prime}\left(h_{2, \varepsilon}\right)\left(\partial_{x}\left(h_{2, \varepsilon}\right)\right)^{2}$ are indeed positive.

The last step is to develop the first two terms on the left hand side of Equation (19) and to use the fact that $2 a b \leq \theta a^{2}+\frac{1}{\theta} b^{2}$ for all $\theta>0$. So, thanks to Gronwall's lemma, the two quantities $\frac{r \nu_{1} g+r \nu_{2} g}{2} \int_{\left\{h_{i, \varepsilon} \leq 1\right\}}\left|\frac{\partial_{x} \varphi_{\varepsilon}\left(h_{i, \varepsilon}\right)}{\sqrt{h_{i, \varepsilon}}}\right|^{2}$ can be controlled, for $i=1,2$.

More precisely, we proved the following result:

Corollary 3.6. The BD mathematical entropy inequality (Equation (19)) 
implies the following bounds:

$$
\begin{gathered}
\left\|\frac{\partial_{x} \varphi_{\varepsilon}\left(h_{i, \varepsilon}\right)}{\sqrt{h_{i, \varepsilon}}}\right\|_{L^{\infty}\left(0, T ; L^{2}(\Omega)\right)}=2\left\|\psi_{\varepsilon}\left(h_{i, \varepsilon}\right) \partial_{x}\left(h_{i, \varepsilon}^{-1 / 2}\right)\right\|_{L^{\infty}\left(0, T ; L^{2}(\Omega)\right)} \leq C(T) \varepsilon, \\
\left\|\varphi_{\varepsilon}^{\prime}\left(h_{i, \varepsilon}\right) \partial_{x} h_{i, \varepsilon}\right\|_{L^{2}\left(0, T ; L^{2}(\Omega)\right)} \leq C(T) .
\end{gathered}
$$

3.2. Uniform bounds for the heights and velocities

Proposition 3.7. Let $\left(h_{1, \varepsilon}, h_{2, \varepsilon}, v_{1, \varepsilon}, v_{2, \varepsilon}\right)$ be a smooth solution of (6)-(9); then for all $T>0$, there exist some constants $\bar{c}(T)>0$ and $\underline{c}(T)>0$ such that

$$
\underline{c}(T) \leq h_{i, \varepsilon}(x, t) \leq \bar{c}(T), \quad \forall x \in \Omega, t \in[0, T], \quad i=1,2 .
$$

The proof of the lower bound relies on two main results. The first one is the following remark:

Remark 3.8. For $\tilde{h}_{i, \varepsilon}$ between fixed reference heights $\left(0<h_{i}^{-}<\tilde{h}_{i, \varepsilon}<h_{i}^{+}\right)$ for $i=1,2$, there exists a constant $K$ such that

$$
\begin{aligned}
& h_{i, \varepsilon}+h_{i, \varepsilon}^{2} \leq K\left(1+\left(h_{i, \varepsilon}-\tilde{h}_{i, \varepsilon}\right)^{2}\right), \\
& \liminf _{h_{i, \varepsilon} \rightarrow 0}\left(h_{i, \varepsilon}-\tilde{h}_{i, \varepsilon}\right)^{2} \geq K^{-1}, \quad i=1,2 .
\end{aligned}
$$

The proof is also related to the following lemma derived in [7] and [15].

Lemma 3.9. For every $T>0$, there exist $\delta>0$ and $R(T)$ such that for every $x_{0} \in \mathbb{R}$ and $t_{0}>0$, there exists $x_{1} \in\left[x_{0}-R(T), x_{0}+R(T)\right]$ with

$$
h_{i, \varepsilon}\left(x_{1}, t_{0}\right)>\delta \text {. }
$$

Let us now detail the proof of Proposition 3.7.

Proof. Let us define $\ell_{i, \varepsilon}=\inf \left(h_{i, \varepsilon}, 1\right)$. We have $\partial_{x} \ell_{i, \varepsilon}=\partial_{x} h_{i, \varepsilon} \mathbf{1}_{\left\{h_{i, \varepsilon} \leq 1\right\}}$. Due to inequality (20), the following bound holds:

$$
\left\|\psi_{\varepsilon}\left(\ell_{i, \varepsilon}\right) \partial_{x}\left(\ell_{i, \varepsilon}^{-1 / 2}\right)\right\|_{L^{\infty}\left(0, T ; L^{2}(\Omega)\right)} \leq C(T) \varepsilon .
$$

Since $\psi_{\varepsilon}\left(\ell_{i, \varepsilon}\right)=h_{i, \varepsilon}+\frac{\varepsilon}{\beta} h_{i, \varepsilon}^{\beta} \geq \frac{\varepsilon}{\beta} h_{i, \varepsilon}^{\beta}=\frac{\varepsilon}{\beta} \ell_{i, \varepsilon}^{\beta}$ in the set where $\left\{h_{i, \varepsilon} \leq 1\right\}$, we deduce that

$$
\frac{\varepsilon}{\beta}\left\|\partial_{x} \ell_{i, \varepsilon}^{\beta-1 / 2}\right\|_{L^{\infty}\left(0, T ; L^{2}(\Omega)\right)} \leq\left\|\psi_{\varepsilon}\left(\ell_{i, \varepsilon}\right) \partial_{x}\left(\ell_{i, \varepsilon}^{-1 / 2}\right)\right\|_{L^{\infty}\left(0, T ; L^{2}(\Omega)\right)} \leq C(T) \varepsilon .
$$


Hence by using the Sobolev-Poincaré inequality, Lemma 3.9 and Equation (24) imply that $\ell_{i, \varepsilon}^{\beta-1 / 2}$ is bounded in $L^{\infty}\left(0, T ; L^{\infty}(\Omega)\right)$. More precisely, we obtain a lower bound for $\ell_{i, \varepsilon}$ (and for $\left.h_{i, \varepsilon}\right): \ell_{i, \varepsilon} \geq(\beta C(T))^{\frac{-2}{1-2 \beta}}$.

Next, we want to get a bound for $\sqrt{h_{i, \varepsilon}}$ in $L^{\infty}\left(0, T ; W^{1,1}(\Omega)\right)$. First, we remark that:

$$
\begin{aligned}
\int_{\Omega}\left|\partial_{x} h_{i, \varepsilon}^{1 / 2}\right| d x & =\frac{1}{2} \int_{\Omega}\left|h_{i, \varepsilon}^{-1 / 2} \partial_{x} h_{i, \varepsilon}\right| d x \\
& \leq\left(\int_{\Omega}\left|h_{i, \varepsilon}\right|\right)^{1 / 2}\left(\int_{\Omega}\left|\frac{\partial_{x} h_{i, \varepsilon}}{h_{i, \varepsilon}}\right|^{2}\right)^{1 / 2} \\
& \leq \frac{1}{2}\left(\int_{\Omega}\left|\sqrt{h_{i, \varepsilon}}\right|^{2} d x\right)^{1 / 2}\left(\int_{\Omega} h_{i, \varepsilon}\left|\frac{\partial_{x} h_{i, \varepsilon}}{\sqrt{h_{i, \varepsilon}}}\right|^{2}\left|\frac{\varphi_{\varepsilon}^{\prime}\left(h_{i, \varepsilon}\right)}{\psi_{\varepsilon}\left(h_{i, \varepsilon}\right)}\right|^{2} d x\right)^{1 / 2} \\
& \leq \frac{1}{2} \frac{1}{\sqrt{\underline{c}(T)}}\left(\int_{\Omega}\left|\sqrt{h_{i, \varepsilon}}\right|^{2} d x\right)^{1 / 2}\left(\int_{\Omega}\left|\frac{\partial_{x} \varphi_{\varepsilon}\left(h_{i, \varepsilon}\right)}{\sqrt{h_{i, \varepsilon}}}\right|^{2}\right)^{1 / 2},
\end{aligned}
$$

as $\psi_{i, \varepsilon} \geq h_{i, \varepsilon}$ and $h_{i, \varepsilon}$ has a lower bound denoted by $\underline{c}(T)$. Thanks to Equation (20), we obtain:

$$
\int_{\Omega}\left|\partial_{x} h_{i, \varepsilon}^{1 / 2}\right| d x \leq \bar{c}(T)
$$

Finally, we have $\partial_{x} \sqrt{h_{i, \varepsilon}} \in L^{\infty}\left(0, T ; L^{1}(\Omega)\right)$ and $\sqrt{h_{i, \varepsilon}} \in L^{\infty}\left(0, T ; L^{2}(\Omega)\right)$; this means that $\sqrt{h_{i, \varepsilon}} \in L^{\infty}\left(0, T ; W^{1,1}(\Omega)\right)$. To conclude, we use Sobolev embeddings to get the upper bound for $h_{i, \varepsilon}$.

Proposition 3.10. For all $T>0$, there exist some constants $\bar{k}_{1}(T), \bar{k}_{2}(T)>$ 0 such that

$$
\left\|v_{i, \varepsilon}\right\|_{L^{2}\left(0, T ; H^{2}(\Omega)\right)} \leq \bar{k}_{1}(T), \quad\left\|\partial_{t} v_{i, \varepsilon}\right\|_{L^{2}\left(0, T ; L^{2}(\Omega)\right)} \leq \bar{k}_{2}(T) .
$$

Proof. Relations (17) and (21) imply that

$$
\left\|v_{i, \varepsilon}\right\|_{L^{\infty}\left(0, T ; L^{2}(\Omega)\right)} \leq C_{1}(T) \text { and }\left\|\partial_{x} v_{i, \varepsilon}\right\|_{L^{2}\left(0, T ; L^{2}(\Omega)\right)} \leq C_{2}(T)
$$

for some positive constants $C_{1}(T), C_{2}(T)$. Moreover, since $v_{i, \varepsilon}$ belongs to $L^{\infty}\left(0, T ; H^{1}(\Omega)\right)$, thanks to Sobolev embeddings, we get:

$$
\partial_{t} h_{i, \varepsilon}=-\partial_{x}\left(h_{i, \varepsilon} v_{i, \varepsilon}\right) \in L^{2}\left(0, T ; L^{2}(\Omega)\right) .
$$


Now let us write the two momentum equations in the following form:

$$
\begin{aligned}
\partial_{t} v_{i, \varepsilon} & -\partial_{x}\left(\frac{\psi_{\varepsilon}\left(h_{i, \varepsilon}\right)}{h_{i, \varepsilon}} \partial_{x} v_{i, \varepsilon}\right) \\
= & -g \partial_{x}\left(h_{i, \varepsilon}+r_{i} h_{j, \varepsilon}\right)-v_{i, \varepsilon} \partial_{x} v_{i, \varepsilon}+\nu_{i} \frac{\partial_{x} h_{i, \varepsilon}}{h_{i, \varepsilon}^{2}} \psi_{\varepsilon}\left(h_{i, \varepsilon}\right) \partial_{x} v_{i, \varepsilon}
\end{aligned}
$$

where $i, j=1,2, i \neq j, r_{1}=r$ and $r_{2}=1$.

It remains to control the right hand side of this equation to deduce a better bound for the velocities $v_{i, \varepsilon}$.

The first term $g \partial_{x}\left(h_{i, \varepsilon}+r_{i} h_{j, \varepsilon}\right)$ is bounded in $L^{2}\left(0, T ; L^{2}(\Omega)\right)$.

Next, we have

$$
\int_{\Omega}\left(\partial_{x} h_{i, \varepsilon}\right)^{2}=\int_{\Omega} \frac{\left(\varphi_{\varepsilon}^{\prime}\left(h_{i, \varepsilon}\right)\right)^{2}\left(\partial_{x} h_{i, \varepsilon}\right)^{2}}{\left(\varphi_{\varepsilon}^{\prime}\left(h_{i, \varepsilon}\right)\right)^{2}} \leq \int_{\Omega} \frac{h_{i, \varepsilon}\left(\partial_{x}\left(\varphi_{\varepsilon}\left(h_{i, \varepsilon}\right)\right)\right)^{2}}{\left(\varepsilon h_{i, \varepsilon}^{\beta-1 / 2}\right)^{2}} .
$$

Due to the BD entropy (20) and relation (21), the last term is bounded. So, $\partial_{x} h_{i, \varepsilon}$ is bounded in $L^{\infty}\left(0, T ; L^{2}(\Omega)\right)$.

Finally, by using Holder and interpolation inequalities, we get:

$$
\begin{aligned}
\|\left(v_{i, \varepsilon}-\right. & \left.\nu_{i} \frac{\partial_{x} h_{i, \varepsilon}}{h_{i, \varepsilon}^{2}} \psi_{\varepsilon}\left(h_{i, \varepsilon}\right)\right) \partial_{x} v_{i, \varepsilon}\left\|_{L^{2}\left(0, T ; L^{4 / 3}(\Omega)\right)}\right\|\left\|_{i, \varepsilon}-\nu_{i} \frac{\partial_{x} h_{i, \varepsilon}}{h_{i, \varepsilon}^{2}} \psi_{\varepsilon}\left(h_{i, \varepsilon}\right)\right\|_{L^{\infty}\left(0, T ; L^{2}(\Omega)\right)}\left\|v_{i, \varepsilon}\right\|_{L^{2}\left(0, T ; L^{4}(\Omega)\right)}, \\
\leq & \left\|v_{i, \varepsilon}-\nu_{i} \frac{\partial_{x} h_{i, \varepsilon}}{h_{i, \varepsilon}^{2}} \psi_{\varepsilon}\left(h_{i, \varepsilon}\right)\right\|_{L^{\infty}\left(0, T ; L^{2}(\Omega)\right)}\left\|\partial_{x} v_{i, \varepsilon}\right\|_{L^{2}\left(0, T ; L^{2}(\Omega)\right)}^{1 / 3}\left\|\partial_{x} v_{i, \varepsilon}\right\|_{L^{2}\left(0, T ; W^{1,4 / 3}(\Omega)\right)}^{2 / 3}, \\
\leq & C(T)\left\|\partial_{x} v_{i, \varepsilon}\right\|_{L^{2}\left(0, T ; W^{1,4 / 3}(\Omega)\right)}^{2 / 3}
\end{aligned}
$$

Hence, thanks to standard regularity results for linear parabolic equations (see [22] for example), we obtain that

$$
\left\|\partial_{x} v_{i, \varepsilon}\right\|_{L^{2}\left(0, T ; W^{1,4 / 3}(\Omega)\right)}^{1 / 3} \leq C(T) .
$$

As a consequence of this bound, we get that the second member of (26) is bounded in $L^{2}\left(0, T ; L^{2}(\Omega)\right)$. So $v_{i, \varepsilon}$ is bounded in $L^{2}\left(0, T ; H^{2}(\Omega)\right)$ and $\partial_{t} v_{i, \varepsilon}$ is bounded in $L^{2}\left(0, T ; L^{2}(\Omega)\right)$. 


\section{Proof of the existence result}

Proposition 4.1. (Solonnikov). Let the functions $\psi_{\varepsilon}\left(h_{i, \varepsilon}\right)$ be lower bounded:

$$
\psi_{\varepsilon}\left(h_{i, \varepsilon}\right) \geq \psi
$$

where $\psi$ is some positive constant, and $\nu_{1}, \nu_{2}$ satisfying relation (11). Assume that the initial conditions $h_{i_{0}, \varepsilon}$ and $v_{i_{0}, \varepsilon}$ satisfy (5), with $\varepsilon$ verifying condition (10). Then there exists $T_{0}>0$ depending on $\underline{c}_{0}, \bar{c}_{0},\left\|h_{i_{0}, \varepsilon}\right\|_{H^{1}(\Omega)}$ and $\left\|v_{i_{0}, \varepsilon}\right\|_{H^{1}(\Omega)}$ such that system (6)-(9) has a unique solution $\left(h_{1, \varepsilon}, h_{2, \varepsilon}, v_{1, \varepsilon}, v_{2, \varepsilon}\right)$ on $\left(0, T_{0}\right)$ satisfying

$$
\begin{array}{ll}
h_{i, \varepsilon} \in L^{\infty}\left(0, T_{1} ; H^{1}(\Omega)\right), & v_{i, \varepsilon} \in L^{2}\left(0, T_{1} ; H^{2}(\Omega)\right), \\
\partial_{t} v_{i, \varepsilon} \in L^{2}\left(0, T_{1} ; L^{2}(\Omega)\right), & \partial_{t} h_{i, \varepsilon} \in L^{2}\left(0, T_{1} ; L^{2}(\Omega)\right),
\end{array}
$$

for all $T_{1}<T_{0}$.

Moreover, there exist some positive constants $\underline{c}(T)$ and $\bar{c}(T)$ such that

$$
\underline{c}(T) \leq h_{i, \varepsilon}(t, x) \leq \bar{c}(T), \quad \forall t \in\left(0, T_{0}\right) .
$$

Proposition 4.2. Let $h_{i_{0}, \varepsilon}$ and $v_{i_{0}, \varepsilon}$ satisfy (5); then the approximate system (6)-(9) admits a strong solution satisfying the energy and entropy inequalities.

Proof. Let us define $\psi_{\varepsilon, n}\left(h_{i, \varepsilon}\right)=\max \left(\psi_{\varepsilon}\left(h_{i, \varepsilon}\right), 1 / n\right)$ for $n \geq 1$; we have $\psi \leq$ $\psi_{\varepsilon, n} \leq \psi+1$. Moreover, for all $n \in \mathbb{N}^{*}, \psi_{\varepsilon, n} \geq \frac{1}{n}>0$. Using Proposition 4.1, we can consider $\left(h_{1, \varepsilon}^{n}, h_{2, \varepsilon}^{n}, v_{1, \varepsilon}^{n}, v_{2, \varepsilon}^{n}\right)$ the strong solution of (6)-(9) where the functions $\psi_{\varepsilon}\left(h_{i, \varepsilon}\right)$ are replaced by $\psi_{\varepsilon, n}\left(h_{i, \varepsilon}\right)$; this solution exists at least for small time $T_{0}$. Due to Proposition $3.7, h_{i, \varepsilon}^{n}$ is uniformly bounded in $n$ from below, and then we can take $T_{0}=\infty$. Assume that $n>1 / \underline{c}(T)$; then $\left(h_{1, \varepsilon}^{n}, h_{2, \varepsilon}^{n}, v_{1, \varepsilon}^{n}, v_{2, \varepsilon}^{n}\right)$ is the solution of $(6)-(9)$ with $\psi_{\varepsilon, n}\left(h_{i, \varepsilon}\right)=\psi_{\varepsilon}\left(h_{i, \varepsilon}\right)$.

Proposition 4.3. Let us define the velocities:

$$
v_{i, \varepsilon}=\frac{\sqrt{h_{i, \varepsilon}} v_{i, \varepsilon}}{\sqrt{h_{i, \varepsilon}}},
$$

which is possible thanks to the lower bound on the heights. The strong solution of the approximate problem (6)-(9) converges to some expected strong solution $\left(h_{1}, h_{2}, v_{1}, v_{2}\right)$ of the problem (1)-(4) as $\varepsilon \rightarrow 0^{+}$in the sense:

$$
\begin{aligned}
& h_{i, \varepsilon} \rightarrow h_{i} \text { in } C([0, T] \times \bar{\Omega}) \\
& v_{i, \varepsilon} \rightarrow v_{i} \text { in } C([0, T] \times \bar{\Omega}) \\
& \psi_{\varepsilon}\left(h_{i, \varepsilon}\right) \rightarrow h_{i} \text { in } C([0, T] \times \bar{\Omega}) \\
& \sqrt{h_{i, \varepsilon}} v_{i, \varepsilon} \rightarrow \sqrt{h_{i}} v_{i} \text { in } L^{2+\theta}(0, T ; \bar{\Omega}),
\end{aligned}
$$


$\theta$ being some positive constant.

Proof. Since we have $\underline{c}(T) \leq h_{i, \varepsilon} \leq \bar{c}(T)$ uniformly with respect to $\varepsilon$, the limit $h_{i}$ is bounded and bounded away from zero. The limit system can then be divided by $h_{i}$ and becomes parabolic with respect to the velocities $v_{i}$. Finally standard regularity results for parabolic systems can be used again to obtain that

$$
\begin{array}{ll}
h_{i} \in L^{\infty}\left(0, T_{1} ; H^{1}(\Omega)\right), & v_{i} \in L^{2}\left(0, T_{1} ; H^{2}(\Omega)\right), \\
\partial_{t} v_{i} \in L^{2}\left(0, T_{1} ; L^{2}(\Omega)\right), & \partial_{t} h_{i} \in L^{2}\left(0, T_{1} ; L^{2}(\Omega)\right) .
\end{array}
$$

\section{Appendix A. Proofs of Propositions 3.2 and 3.4}

This section is devoted to the proofs of the energy and entropy inequalities used in Section 3. For the classical energy, as usual, the idea is to multiply the momentum equation by the velocity. More precisely, we multiply the momentum equations $(7)$ and (9) by $v_{1, \varepsilon}$ and $v_{2, \varepsilon}$ respectively, and we integrate by parts. For $i=1,2$, we obtain:

$$
\begin{array}{r}
\int_{\Omega}\left(\partial_{t}\left(h_{1, \varepsilon} v_{1, \varepsilon}\right)+\partial_{x}\left(h_{1, \varepsilon} v_{1, \varepsilon}^{2}\right)\right) \\
v_{1, \varepsilon} d x+g \int_{\Omega}\left(h_{1, \varepsilon} \partial_{x} h_{1, \varepsilon}+r h_{1, \varepsilon} \partial_{x} h_{2, \varepsilon}\right) v_{1, \varepsilon} d x \\
\left.-\nu_{1} \int_{\Omega} \partial_{x}\left(\psi_{\varepsilon}\left(h_{1, \varepsilon}\right) \partial_{x}\left(v_{1, \varepsilon}\right)\right) v_{1, \varepsilon} d x=0, \quad \text { (A. } 1\right)
\end{array}
$$

and

$$
\begin{array}{r}
\int_{\Omega}\left(\partial_{t}\left(h_{2, \varepsilon} v_{2, \varepsilon}\right)+\partial_{x}\left(h_{2, \varepsilon} v_{2, \varepsilon}^{2}\right)\right) \\
v_{2, \varepsilon} d x+g \int_{\Omega}\left(h_{2, \varepsilon} \partial_{x} h_{2, \varepsilon}+h_{2, \varepsilon} \partial_{x} h_{1, \varepsilon}\right) v_{2, \varepsilon} d x \\
-\nu_{2} \int_{\Omega} \partial_{x}\left(\psi_{\varepsilon}\left(h_{2, \varepsilon}\right) \partial_{x}\left(v_{2, \varepsilon}\right)\right) v_{2, \varepsilon} d x=0 . \quad \text { (A.2) }
\end{array}
$$

We can reformulate some terms, namely (for $i=1,2$ )

$$
\begin{aligned}
\int_{\Omega}\left(\partial_{t}\left(h_{i, \varepsilon} v_{i, \varepsilon}\right)+\partial_{x}\left(h_{i, \varepsilon} v_{i, \varepsilon}^{2}\right)\right) v_{i, \varepsilon} d x & =\frac{1}{2} \frac{d}{d t} \int_{\Omega} h_{i, \varepsilon}\left|v_{i, \varepsilon}\right|^{2} d x \\
-\nu_{i} \int_{\Omega} \partial_{x}\left(\psi_{\varepsilon}\left(h_{i, \varepsilon}\right) \partial_{x}\left(v_{i, \varepsilon}\right)\right) v_{i, \varepsilon} d x & =\nu_{i} \int_{\Omega} \psi_{\varepsilon}\left(h_{i, \varepsilon}\right)\left(\partial_{x}\left(v_{i, \varepsilon}\right)\right)^{2} d x
\end{aligned}
$$


To obtain the energy inequality, we add (A.1) to (A.2) multiplied by $r$. We remark that:

$$
\begin{aligned}
& g \int_{\Omega} h_{1, \varepsilon} \partial_{x} h_{1, \varepsilon} v_{1, \varepsilon} d x+r g \int_{\Omega} h_{1, \varepsilon} \partial_{x} h_{2, \varepsilon} v_{1, \varepsilon} d x+r g \int_{\Omega} h_{2, \varepsilon} \partial_{x} h_{2, \varepsilon} v_{2, \varepsilon} d x \\
& \quad+r g \int_{\Omega} h_{2, \varepsilon} \partial_{x} h_{1, \varepsilon} v_{2, \varepsilon} d x=\frac{g(1-r)}{2} \frac{d}{d t} \int_{\Omega}\left|h_{1, \varepsilon}\right|^{2}+\frac{r g}{2} \frac{d}{d t} \int_{\Omega}\left|h_{1, \varepsilon}+h_{2, \varepsilon}\right|^{2} .
\end{aligned}
$$

Thanks to this simplification, we obtain the classical energy estimate (16).

The second proof of this section is that for the BD entropy estimate (18). Multiplying the mass equations by $\varphi_{\varepsilon}^{\prime}\left(h_{i, \varepsilon}\right)$ (for $i=1,2$ ), we get

$$
\partial_{t} \varphi_{\varepsilon}\left(h_{i, \varepsilon}\right)+\partial_{x}\left(\varphi_{\varepsilon}\left(h_{i, \varepsilon}\right)\right) v_{i, \varepsilon}+\varphi_{\varepsilon}^{\prime}\left(h_{i, \varepsilon}\right) h_{i, \varepsilon} \partial_{x} v_{i, \varepsilon}=0 .
$$

Thanks to Equation (13), this relation reads

$$
\partial_{t} \varphi_{\varepsilon}\left(h_{i, \varepsilon}\right)+\partial_{x}\left(\varphi_{\varepsilon}\left(h_{i, \varepsilon}\right)\right) v_{i, \varepsilon}+\psi_{\varepsilon}\left(h_{i, \varepsilon}\right) \partial_{x} v_{i, \varepsilon}=0 .
$$

We differentiate this equation with respect to $x$ and introduce the corresponding viscosity coefficient to get

$$
\begin{aligned}
\partial_{t}\left(h_{i, \varepsilon} \nu_{i} \frac{\partial_{x}\left(\varphi_{\varepsilon}\left(h_{i, \varepsilon}\right)\right)}{h_{i, \varepsilon}}\right)+\partial_{x}\left(h_{i, \varepsilon} \nu_{i} \frac{\partial_{x}\left(\varphi_{\varepsilon}\left(h_{i, \varepsilon}\right)\right)}{h_{i, \varepsilon}} v_{i, \varepsilon}\right) \\
+\nu_{i} \partial_{x}\left(\psi_{\varepsilon}\left(h_{i, \varepsilon}\right) \partial_{x}\left(v_{i, \varepsilon}\right)\right)=0 .
\end{aligned}
$$

We add the momentum equation (Equation (7) for $i=1$ and Equation (9) for $i=2$ ) to the previous equality and we get

$$
\begin{aligned}
\partial_{t}\left(h_{1, \varepsilon} v_{1, \varepsilon}+h_{1, \varepsilon} \nu_{1} \frac{\partial_{x} \varphi_{\varepsilon}\left(h_{1, \varepsilon}\right)}{h_{1, \varepsilon}}\right)+ & \partial_{x}\left(h_{1, \varepsilon} v_{1, \varepsilon}^{2}+h_{1, \varepsilon} \nu_{1} \frac{\partial_{x} \varphi_{\varepsilon}\left(h_{1, \varepsilon}\right)}{h_{1, \varepsilon}} v_{1, \varepsilon}\right) \\
+ & g h_{1, \varepsilon} \partial_{x} h_{1, \varepsilon}+r g h_{1, \varepsilon} \partial_{x} h_{2, \varepsilon}=0, \\
\partial_{t}\left(h_{2, \varepsilon} v_{2, \varepsilon}+h_{2, \varepsilon} \nu_{2} \frac{\partial_{x} \varphi_{\varepsilon}\left(h_{2, \varepsilon}\right)}{h_{2, \varepsilon}}\right)+\partial_{x} & \left(h_{2, \varepsilon} v_{2, \varepsilon}^{2}+h_{2, \varepsilon} \nu_{2} \frac{\partial_{x} \varphi_{\varepsilon}\left(h_{2, \varepsilon}\right)}{h_{2, \varepsilon}} v_{2, \varepsilon}\right) \\
& +g h_{2, \varepsilon} \partial_{x} h_{2, \varepsilon}+g h_{2, \varepsilon} \partial_{x} h_{1, \varepsilon}=0 .
\end{aligned}
$$

We add Equation (A.5) multiplied by $\left(v_{1, \varepsilon}+\nu_{1} \frac{\partial_{x}\left(\varphi_{\varepsilon}\left(h_{1, \varepsilon}\right)\right)}{h_{1, \varepsilon}}\right)$ to Equation (A.6) multilplied by $r\left(v_{2, \varepsilon}+\nu_{2} \frac{\partial_{x}\left(\varphi_{\varepsilon}\left(h_{2, \varepsilon}\right)\right)}{h_{2, \varepsilon}}\right)$. Integrating over $\Omega$, we obtain the BD entropy (18). 


\section{References}

[1] G. Narbona-Reina, J. D. D. Zabsonré, E. Fernández-Nieto and D. BREsCH. Derivation of a Bilayer Model for Shallow Water Equations with Viscosity. Numerical Validation, CMES, 43(1), 27-71 (2009).

[2] E. Audusse. A multilayer Saint-Venant model: Derivation and numerical validation, Discrete Contin. Dyn. Syst. Ser. B, 5(2), 189-214 (2005).

[3] M. J. Castro, J. Macías, C. Parés, J. A. García-Rodríguez and E. VÁzquez-CEndón. A two-layer finite volume model for flows through channels with irregular geometry: Computation of maximal exchange solutions: Applications to the Strait of Gibraltar, Commun. Nonlinear Sci. Numer. Simul., 9(2), 241-249 (2004).

[4] A. Kazhikhov and V. Shelukhin. Unique global solution with respect to time of initial-boundary value problems for one-dimensional equations of a viscous gas, (translated from Prikl. Mat. Mekh., 41(2), 282-291 (1977)), J. Appl. Math. Mech., 41(2), 273-282 (1977).

[5] D. Hoff. Global existence for 1D, compressible, isentropic NavierStokes equations with large initial data, Trans. Amer. Math. Soc., 303(1), 169-181 (1987).

[6] D. Hoff. Global Solutions of the Navier-Stokes Equations for Multidimensional Compressible Flow with Discontinuous Initial Data, J. Differential Equations, 120(1), 215-254 (1995).

[7] D. Hoff. Global solutions of the equations of one-dimensional, compressible flow with large data and forces, and with differing end states, Z. Angew. Math. Phys., 49(5), 774-785 (1998).

[8] D. Serre. Solutions faibles globales des équations de Navier-Stokes pour un fluide compressible, C. R. Acad. Sci. Paris Sér. I Math., 303(13), 639-642 (1986).

[9] P.-L Lions. Mathematical Topics in Fluid Mechanics. Vol. 2, Compressible Models. Clarendon Press. Oxford (1998).

[10] E. FeIREISL. On the motion of a viscous, compressible, and heat conducting fluid, Indiana Univ. Math. J., 53(6), 1707-1740 (2004). 
[11] D. Bresch, B. Desjardins and C. K. Lin, On Some Compressible Fluids Models: Korteweg, Lubrication and Shallow Water Systems, Commun. Partial Diff. Equations, 28(3-4), 843-868 (2003).

[12] D. Bresch and B. Desjardins. Existence of Global Weak Solutions for a 2D Viscous Shallow Water Equations and Convergence to the Quasi-Geostrophic Model, Comm. Math. Phys., 238(1-2), 211-223 (2003).

[13] D. Bresch and B. Desjardins. On the construction of approximate solutions for the 2D viscous shallow water model and for compressible Navier-Stokes models, J. Maths Pures Appl., 86(4), 362-368 (2006).

[14] D. Bresch and B. Desjardins. On the existence of global weak solutions to the Navier-Stokes equations for viscous compressible and heat conducting fluids, J. Math. Pures Appl., 87(1), 57-90 (2007).

[15] A. Mellet and A. Vasseur. Existence and Uniqueness of Global Strong Solutions for One-Dimensional Compressible Navier-Stokes Equations, SIAM, J. Math. Anal., 39(4), 1344-1365 (2008).

[16] A. Mellet and A. Vasseur. On the Barotropic Compressible NavierStokes Equations, Comm. Partial Diff. Eqs, 32(3), 431-452 (2007).

[17] M. L. Muñoz-Ruiz, M. J. Castro-Díaz and C. Parés . On an onedimensional bi-layer shallow-water problem. Nonlinear Analysis, 53(5), 567-600 (2003).

[18] G. Narbona-Reina and J. D. D. Zabsonré. Existence of global weak solutions for a viscous 2D bilayer Shallow Water model, Comptes Rendus Mathematique, 349(5-6), 285-289 (2011).

[19] J. D. D. Zabsonré and G. Narbona-Reina. Existence of a global weak solution for a 2D viscous bi-layer Shallow Water model, Nonlin. Anal., Real World Appl., 10(5), 2971-2984 (2009).

[20] H.-L. LI, J. Li and Z. Xin. Vanishing of Vacuum States and Blowup Phenomena of the Compressible Navier-Stokes Equations, Commun. Math. Phys., 281(2), 401-444 (2008). 
[21] S. Jiang, Z. Xin and P. Zhang. Global weak solutions to 1D compressible isentropic Navier-Stokes equations with density-dependent viscosity, Methods and Applications of Analysis, 12(3), 239-252 (2005).

[22] O. A. Ladyzenskaja, V. A. Solonnikov and N.N. Uraltseva. Linear and quasilinear equations of parabolic type, translated from the Russian by S. Smith. Translations of Mathematical Monographs, Vol. 23. Providence, R.I. American Mathematical Society (1968).

[23] Z. Guo, Q. Jiu and Z. XIN. Spherically Symmetric Isentropic Compressible Flows with Density Dependent Viscosity Coefficients, SIAM J. Math. Anal., 39(5), 1402-1427 (2008). 